\title{
WEYL FORMULA FOR THE EIGENVALUES OF THE DISSIPATIVE ACOUSTIC OPERATOR
}

\author{
VESSELIN PETKOV
}

\begin{abstract}
We study the wave equation in the exterior of a bounded domain $K$ with dissipative boundary condition $\partial_{\nu} u-\gamma(x) \partial_{t} u=0$ on the boundary $\Gamma$ and $\gamma(x)>0$. The solutions are described by a contraction semigroup $V(t)=e^{t G}, t \geq 0$. The eigenvalues $\lambda_{k}$ of $G$ with $\operatorname{Re} \lambda_{k}<0$ yield asymptotically disappearing solutions $u(t, x)=e^{\lambda_{k} t} f(x)$ having exponentially decreasing global energy. We establish a Weyl formula for these eigenvalues in the case $\min _{x \in \Gamma} \gamma(x)>1$. For strictly convex obstacles $K$ this formula concerns all eigenvalues of $G$.
\end{abstract}

Keywords: Dissipative boundary conditions, eigenvalues asymptotics

Mathematics Subject Classification 2020: 35P20, 35P25, 47A40, 58J50

\section{INTRODUCTION}

Let $K \subset \mathbb{R}^{d}, d \geq 2$, be a bounded non-empty domain. Let $\Omega=\mathbb{R}^{d} \backslash \bar{K}$ be connected. and $K \subset\left\{x \in \mathbb{R}^{d}:|x| \leq \rho_{0}\right\}$. We suppose that the boundary $\Gamma$ of $K$ is $C^{\infty}$. Consider the boundary problem

$$
\left\{\begin{array}{l}
u_{t t}-\Delta_{x} u=0 \text { in } \mathbb{R}_{t}^{+} \times \Omega, \\
\partial_{\nu} u-\gamma(x) \partial_{t} u=0 \text { on } \mathbb{R}_{t}^{+} \times \Gamma, \\
u(0, x)=f_{1}, u_{t}(0, x)=f_{2}
\end{array}\right.
$$

with initial data $\left(f_{1}, f_{2}\right) \in H^{1}(\Omega) \times L^{2}(\Omega)=\mathcal{H}$. Here $\nu(x)$ is the unit outward normal to $\Gamma$ pointing into $\Omega$ and $\gamma(x) \geq 0$ is a $C^{\infty}$ function on $\Gamma$. The solution of the problem (1.1) is given by $V(t) f=e^{t G} f, t \geq 0$, where $V(t)$ is a contraction semi-group in $\mathcal{H}$ whose generator

$$
G=\left(\begin{array}{cc}
0 & 1 \\
\Delta & 0
\end{array}\right)
$$

has a domain $D(G)$ which is the closure in the graph norm

$$
|\|f\||=\left(\|f\|_{\mathcal{H}}^{2}+\|G f\|_{\mathcal{H}}^{2}\right)^{1 / 2}
$$

of functions $f=\left(f_{1}, f_{2}\right) \in C_{(0)}^{\infty}\left(\mathbb{R}^{d}\right) \times C_{(0)}^{\infty}\left(\mathbb{R}^{d}\right)$ satisfying the boundary condition $\partial_{\nu} f_{1}-\gamma f_{2}=$ 0 on $\Gamma$. It is well known that the spectrum of $G$ in $\operatorname{Re} z<0$ is formed by isolated eigenvalues with finite multiplicity (see [7] for $d$ odd and [12] for all $d \geq 2$.) Moreover, $G$ has no eigenvalues on the imaginary axis $\mathbf{i} \mathbb{R}$. Notice that if $G f=\lambda f$ with $0 \neq f \in D(G), \operatorname{Re} \lambda<0$ and $\partial_{\nu} f_{1}-\gamma f_{2}=0$ on $\Gamma$, we get

$$
\left\{\begin{array}{l}
\left(\Delta-\lambda^{2}\right) f_{1}=0 \text { in } \Omega \\
\partial_{\nu} f_{1}-\lambda \gamma f_{1}=0 \text { on } \Gamma
\end{array}\right.
$$

e-mail:petkov@math.u-bordeaux.fr. 
and $u(t, x)=V(t) f=e^{\lambda t} f(x)$ is a solution of (1.1) with exponentially decreasing global energy. Such solutions are called asymptotically disappearing. On the other hand, the solutions $u(t, x)=V(t) f$ for which there exists $T>0$ such that $u(t, x) \equiv 0$ for $t \geq T$ are called disappearing (see [8]). For $t_{0}>0$ the closed linear space

$$
H\left(t_{0}\right)=\left\{g \in \mathcal{H}: V(t) g=0 \text { for } t \geq t_{0}\right\}
$$

is invariant under the action of $V(t)$ and if $H\left(t_{0}\right) \neq\{0\}$, then $H\left(t_{0}\right)$ has infinite dimension. If $H\left(t_{0}\right)$ is not trivial, the scattering system is non controllable (see section 4 in 8 for the definition and details). Majda proved in [8] that for obstacles with analytic boundary $\Gamma$ and analytic $\gamma(x)$ the condition $\gamma(x) \neq 1, \forall x \in \Gamma$, implies that there are no disappearing solutions.

In this paper in the case $\min _{x \in \Gamma} \gamma(x)>1$ we show that there exists a subspace $\mathcal{H}_{s p} \subsetneq$ $\mathcal{H}$ with infinite dimension generated by eigenfunctions of $G$ such that $V(t) g, g \in \mathcal{H}_{s p}$ is asymptotically disappearing. The eigenvalues $\lambda_{k}$ sufficiently close to $\mathbb{R}^{-}$with $\operatorname{Re} \lambda_{k} \rightarrow-\infty$ present a particular interest for applications since they correspond to solutions decreasing sufficiently fast as $t \rightarrow+\infty$. It is important to know that such eigenvalues exist and to have their asymptotic. It was proved in [2] that if we have at least one eigenvalue $\lambda$ of $G$ with $\operatorname{Re} \lambda<0$, then the wave operators $W_{ \pm}$are not complete, that is $\operatorname{Ran} W_{-} \neq \operatorname{Ran} W_{+}$. Hence we cannot define the scattering operator $S$ related to the Cauchy problem for the free wave equations and the boundary problem (1.1) by the product $W_{+}^{-1} \circ W_{-}$. When the global energy is conserved in time and the unperturbed and perturbed problems are associated to unitary groups, the corresponding scattering operator $S(z): L^{2}\left(\mathbb{S}^{d-1}\right) \rightarrow L^{2}\left(\mathbb{S}^{d-1}\right)$ satisfies the identity

$$
S^{-1}(z)=S^{*}(\bar{z}), z \in \mathbb{C}
$$

providing $S(z)$ invertible at $z$. Since $S(z)$ and $S^{*}(z)$ are analytic in the "physical" half plane $\{z \in \mathbb{C}: \operatorname{Im} z<0\}$ (see [6]) the above relation implies that $S(z)$ is invertible for $\operatorname{Im} z>0$. For dissipative boundary problems the relation (1.3) in general is not true and $S\left(z_{0}\right)$ may have a non trivial kernel for some $z_{0}, \operatorname{Im} z_{0}>0$. For odd dimensions $d$ Lax and Phillips [7] proved that this implies that $\mathbf{i} z_{0}$ is an eigenvalue of $G$. Thus the analysis of the eigenvalues of $G$ is important for the location and the existence of points, where the kernel of $S(z)$ is not trivial. A similar connection occurs in the analysis of the interior transmission eigenvalues (see [1] for the definition and more references). More precisely, consider the far-filed operator

$$
(F(k) f)(\theta)=\int_{\mathbb{S}^{d-1}} a(k, \theta, \omega) f(\omega) d \omega,(\theta, \omega) \in \mathbb{S}^{d-1} \times \mathbb{S}^{d-1} .
$$

Here $a(k, \theta, \omega)$ is the scattering amplitude for the Helmholtz equation $\left(\Delta+k^{2} n(x)\right) u=$ $0, x \in K$ with contrast function $n(x)>0$ and for $d$ odd the scattering operator has the representation

$$
S(k)=I d+\left(\frac{\mathbf{i} k}{2 \pi}\right)^{(d-1) / 2} F(k), k \in \mathbb{R} .
$$

Therefore if the kernel of $F(k)$ is non trivial, $k$ is an interior transmission eigenvalue [1].

The location in $\mathbb{C}$ of the eigenvalues of $G$ has been studied in [12] improving previous results of Majda [9]. It was proved in [12 that for the case when $K$ is the unit ball $B_{3}=$ $\left\{x \in \mathbb{R}^{3}:|x| \leq 1\right\}$ and $\gamma \equiv 1$, the operator $G$ has no eigenvalues. For this reason we study the cases

$$
(A): \max _{x \in \Gamma} \gamma(x)<1,(B): \min _{x \in \Gamma} \gamma(x)>1
$$


The results in [12] say that in the case (B) for every $0<\epsilon \ll 1$ and every $M \in \mathbb{N}, M \geq 1$ the eigenvalues lie in $\Lambda_{\epsilon} \cup \mathcal{R}_{N}$, where

$$
\begin{gathered}
\Lambda_{\epsilon}=\left\{z \in \mathbb{C}:|\operatorname{Re} z| \leq C_{\epsilon}\left(1+|\operatorname{Im} z|^{1 / 2+\epsilon}\right), \operatorname{Re} z<0\right\} \\
\mathcal{R}_{M}=\left\{z \in \mathbb{C}:|\operatorname{Im} z| \leq A_{M}(1+|\operatorname{Re} z|)^{-M}, \operatorname{Re} z<0\right\} .
\end{gathered}
$$

Moreover, for strictly convex obstacles $K$ there exists $R_{0}>0$ such that the eigenvalues lie in $\mathcal{R}_{M} \cup\left\{|z| \leq R_{0}\right\}$. In the case (A) the eigenvalues lie in $\Lambda_{\epsilon}$. By using the results in [18], it is possible to improve the eigenvalue free regions replacing $\Lambda_{\epsilon}$ by $\left\{z \in \mathbb{C}:-A_{0} \leq \operatorname{Re} z<0\right\}$ with sufficiently large $A_{0}>0$.

The existence of eigenvalues has been proved (see Appendix in [12]) only for the ball $B_{3}$ and $\gamma \equiv$ const $>1$ and in this particular case we have

$$
\sigma_{p}(G) \subset\left(-\infty,-\frac{1}{\gamma-1}\right] .
$$

Moreover, we have infinite number of real eigenvalues and as $\gamma \searrow 1$ one gets a large strip $\left\{z \in \mathbb{C}:-\frac{1}{\gamma-1}<\operatorname{Re} z<0\right\}$ without eigenvalues.

The purpose of this paper is to establish a Weyl formula for the eigenvalues in $\mathcal{R}_{M} \cap\{z \in$ $\left.\mathbb{C}: \operatorname{Re} z<-C_{0} \leq-1\right\}$ in the case (B). Introduce the set

$$
\Lambda=\left\{\lambda \in \mathbb{C}:|\operatorname{Im} \lambda| \leq C_{1}(1+|\operatorname{Re} \lambda|)^{-2}, \operatorname{Re} \lambda \leq-C_{0} \leq-1\right\}
$$

containing $\mathcal{R}_{M}, \forall M \geq 2$, modulo a compact set and denote by $\sigma_{p}(G)$ the point spectrum of $G$. Increasing the constant $C_{0}>0$ in the definition of $\Lambda$, we subtract a compact set and this is not important for the asymptotic (1.5) below. In the following we assume that $C_{0} \geq 2 C_{1}$. Given $\lambda \in \sigma_{p}(G)$, we define the algebraic multiplicity of $\lambda$ by

$$
\operatorname{mult}(\lambda)=\operatorname{tr} \frac{1}{2 \pi \mathbf{i}} \int_{|z-\lambda|=\epsilon}(z-G)^{-1} d z
$$

with $0<\epsilon \ll 1$ sufficiently small. Our main result is the following

Theorem 1. Assume $\gamma(x)>1$ for all $x \in \Gamma$. Then the counting function of the eigenvalues in $\Lambda$ taken with their multiplicities has the asymptotic

$$
\begin{array}{r}
\sharp\left\{\lambda_{j} \in \sigma_{p}(G) \cap \Lambda:\left|\lambda_{j}\right| \leq r, r \geq C_{\gamma}\right\} \\
=\frac{\omega_{d-1}}{(2 \pi)^{d-1}}\left(\int_{\Gamma}\left(\gamma^{2}(x)-1\right)^{(d-1) / 2} d S_{x}\right) r^{d-1}+\mathcal{O}_{\gamma}\left(r^{d-2}\right), r \rightarrow \infty,
\end{array}
$$

$\omega_{d-1}$ being the volume of the unit ball $\left\{x \in \mathbb{R}^{d-1}:|x| \leq 1\right\}$.

The example concerning the ball $B_{3}$ and (1.4) show that the condition $r \geq C_{\gamma}$ is natural since the coefficient before $r^{d-1}$ in (1.5) goes to 0 as $\max _{x \in \Gamma} \gamma(x) \searrow 1$. Notice that for strictly convex obstacles $K$ in the case (B) we obtain a Weyl formula for all eigenvalues of $G$. For Maxwell's equations with dissipative boundary conditions in the particular case $K=B_{3}, \gamma \equiv$ const $\neq 1$, the formula (1.5) has been obtained in [4]. Weyl formula for the transmission eigenvalues have been obtained by several authors. We refer to [13] and [11] for more references. It is important to note that in [13 the Weyl formula is established with remainder which depends on the eigenvalue free region. In [11] the relation with the eigenvalues free regions is not exploited and the argument is based on a Tauberian theorem which yields a weak remainder. In the present paper we apply the eigenvalue free results in [12] and the remainder in (1.5) is optimal. 
To prove Theorem 1, we apply the approach of [15] and the construction of a semiclassical parametrix $T(h, z), 0<h \leq h_{0}, z=-\frac{1}{(1+\mathbf{i} \eta)^{2}},|\eta| \leq h^{2}$ for the semi-classical exterior Dirichlet-to-Neumann map $N(h, z)$ given in [17], [12]. For $z=-1$ the operator $P(h):=T(h,-1)-\gamma(x)$ is self-adjoint and we denote by $\mu_{1}(h) \leq \mu_{2}(h) \leq \ldots$ its eigenvalues counted with their multiplicities. The points $0<h_{k} \leq h_{0}$ for which $\mu_{k}\left(h_{k}\right)=0$ correspond to points $h$ for which $P(h)$ is not invertible. For large fixed $k_{0}$, depending on $h_{0}$, the eigenvalues $\mu_{k}\left(h_{0}\right)$ are positive, whenever $k>k_{0}$. Thus if $\mu_{k}\left(r^{-1}\right)<0, k>k_{0}$, we have $\mu_{k}\left(h_{k}\right)=0$ for some $r^{-1}<h_{k}<h_{0}$ and by a more fine analysis we prove that such a $h_{k}$ is unique. The operator $P(h)$ can be extended as holomorphic one for complex $\tilde{h}=h(1+\mathbf{i} \eta) \in L$ with $|\eta| \leq h^{2}$ and $L$ defined in (2.12). For the resolvent $(\lambda-G)^{-1}$ a trace formula has been established in [12] (see Proposition 1). Similarly, a trace formula involving $P^{-1}(\tilde{h})$ and the derivative $\dot{P}(\tilde{h})$ can be proved. These two trace formulas differs by negligible terms and this leads to a map between the points $h_{k} \in L$, where $P\left(h_{k}\right)$ is not invertible and the eigenvalues of $G$. To obtain (1.5), one counts the number of the negative eigenvalues of $P\left(r^{-1}\right), r \geq C_{\gamma}$ which is given by well known formula.

The analysis of the counting function of the eigenvalues of $G$ lying in a strip $\{z \in \mathbb{C}$ : $\left.-A_{0} \leq \operatorname{Re} z \leq 0\right\}, A_{0}>0$, as well as the study of the case $(A)$ are open problems. There is a conjecture that there exists a sequence of eigenvalues $\lambda_{k},\left|\operatorname{Im} \lambda_{k}\right| \rightarrow \infty$. For the investigation of these problems it seems convenient to use the semi-classical parametrix $T(h, z)$ for the exterior Dirichlet-to-Neumann problem constructed in [16] for strictly convex obstacles in the hyperbolic region $\{z \in \mathbb{C}: z=1+\mathbf{i} h w\},|w| \leq B_{0}$.

The paper is organised as follows. In Section 2 we collect some facts concerning the operator $\mathcal{C}(\lambda)=\mathcal{N}(\lambda)-\lambda \gamma$ for $\operatorname{Re} \lambda<0$, where $\mathcal{N}(\lambda)$ is exterior Dirichlet-to-Neumann map defined in the beginning of Section 2. We recall a the trace formula involving the resolvent $(G-\lambda)^{-1}$ established in [12]. In Section 3 one presents some information for the semi-classical parametrix for $N(h, z)$ and $z \in Z_{e}=\left\{z \in \mathbb{C}: z=-\frac{1}{(1+\mathbf{i} \eta)^{2}}\right\},|\eta| \leq h^{2}$ based on the construction in [17], [19]. The properties of the operator $P(h)$ for $h$ real are treated in Section 4. In Section 5 we compare the trace formulas for $\mathcal{C}(\lambda)$ and for $P(\tilde{h})$ and we prove Theorem 1. Finally, in Section 6 we discuss some generalisations and a dissipative boundary problem for Maxwell's equations.

\section{Preliminaries}

We start with some facts which are necessary for our exposition (see [12]). For $\operatorname{Re} \lambda<0$ introduce the exterior Dirichlet-to-Neumann map

$$
\mathcal{N}(\lambda):\left.H^{s}(\Gamma) \ni f \longrightarrow \partial_{\nu} u\right|_{\Gamma} \in H^{s-1}(\Gamma)
$$

where $u$ is the solution of the problem

$$
\left\{\begin{array}{l}
\left(-\Delta+\lambda^{2}\right) u=0 \text { in } \Omega, u \in H^{2}(\Omega), \\
u=f \text { on } \Gamma \\
u:(\mathbf{i} \lambda)-\text { outgoing. }
\end{array}\right.
$$

A function $u(x)$ is $(\mathbf{i} \lambda)$-outgoing if there exists $R>\rho_{0}$ and $g \in L_{\text {comp }}^{2}\left(\mathbb{R}^{d}\right)$ such that

$$
u(x)=\left(-\Delta_{0}+\lambda^{2}\right)^{-1} g,|x| \geq R,
$$

where $R_{0}(\lambda)=\left(-\Delta_{0}+\lambda^{2}\right)^{-1}$ is the outgoing resolvent of the free Laplacian $-\Delta_{0}$ in $\mathbb{R}^{d}$ which is analytic in $\mathbb{C}$ for $d$ odd and on the logarithmic covering of $\mathbb{C}$ for $d$ even. The resolvent 
$R_{0}(\lambda)$ has kernel

$$
R_{0}(\lambda, x-y)=-\left.\frac{\mathbf{i}}{4}\left(\frac{-\mathbf{i} \lambda}{2 \pi|x-y|}\right)^{(n-2) / 2}\left(H_{\frac{n-2}{2}}^{(1)}(u)\right)\right|_{u=-\mathbf{i} \lambda|x-y|},
$$

$H_{\nu}^{(1)}(z)$ being the Hankel function of first kind and we have the asymptotic

$$
H_{\nu}^{(1)}(z)=\left(\frac{2}{\pi r}\right)^{1 / 2} e^{\mathbf{i}\left(z-\frac{\nu \pi}{2}-\frac{\pi}{4}\right)}+\mathcal{O}\left(r^{-3 / 2}\right),-\pi<\arg z<2 \pi,|z|=r \rightarrow+\infty .
$$

The solution of the problem (2.1) with $f \in H^{3 / 2}(\Gamma)$ has the representation

$$
u=e(f)+\left(-\Delta_{D}+\lambda^{2}\right)^{-1}\left(\left(\Delta-\lambda^{2}\right)(e(f)),\right.
$$

where $e(f): H^{3 / 2}(\Gamma) \ni f \rightarrow e(f) \in H_{\text {comp }}^{2}(\Omega)$ is an extension operator and $R_{D}(\lambda)=$ $\left(-\Delta_{D}+\lambda^{2}\right)^{-1}$ is the outgoing resolvent of the Dirichlet Laplacian $\Delta_{D}$ in $\Omega$. The cut-off resolvent $R_{\chi}(\lambda)=\chi(x) R_{D}(\lambda) \chi(x)$ with $\chi(x) \in C_{0}^{\infty}\left(\mathbb{R}^{d}\right)$ equal to 1 in a neighbourhood of $K \cup \operatorname{supp} e(f)$ is analytic for $\operatorname{Re} \lambda<0$ and meromorphic in $\mathbb{C}$ for $d$ odd and on the logarithmic covering of $\mathbb{C}$ for $d$ even. Consequently, $\mathcal{N}(\lambda): H^{3 / 2}(\Gamma) \rightarrow H^{1 / 2}(\Gamma)$ is a meromorphic operator-valued function with the same poles as $R_{\chi}(\lambda)$. The same result holds for the action of $\mathcal{N}(\lambda)$ on other Sobolev spaces. Consider the set $\Lambda \subset\left\{z \in \mathbb{C}: \operatorname{Re} z<-C_{0} \leq-1\right\}$ introduced in Section 1. By using the estimates for $R_{\chi}(\lambda)$ for $\operatorname{Re} \lambda<-C_{0}$, we obtain

$$
\|\mathcal{N}(\lambda)\|_{H^{1 / 2}(\Gamma) \rightarrow H^{-1 / 2}(\Gamma)} \leq A_{0}|\lambda|^{2}, \lambda \in \Lambda
$$

Applying Green's representation for the solution $u(y)$ of (2.1) and taking the limit

$$
\Omega \ni y_{n} \rightarrow x \in \Gamma
$$

we have

$$
\left(C_{00}(\lambda) f\right)(x)-\left(C_{01}(\lambda) \mathcal{N}(\lambda) f\right)(x)=\frac{f(x)}{2}, x \in \Gamma
$$

where

$$
\begin{gathered}
\left(C_{00}(\lambda) f\right)(x)=\int_{\Gamma} f(y) \frac{\partial}{\partial \nu(y)} R_{0}(\lambda, x-y) d S_{y} \\
\left(C_{01}(\lambda) g\right)(x)=\int_{\Gamma} g(y) R_{0}(\lambda, x-y) d S_{y}
\end{gathered}
$$

are the Calderón operators or double and single layer potentials which have the same analytic properties as $R_{0}(\lambda, x-y)$. Melrose showed ([10], Section 3) that there exists an entire family $P_{D}(\lambda)$ of compact pseudo-differential operators of order -1 on $\Gamma$ such that

$$
-2\left(-\Delta_{\Gamma}+1\right)^{1 / 2} C_{01}(\lambda)=I d+P_{D}(\lambda)
$$

$\Delta_{\Gamma}$ being the Laplace Beltrami operator on $\Gamma$ equipped with the Riemannian metric induced by the Euclidean one in $\mathbb{R}^{d}$. In fact, $-C_{01}(\lambda)$ is a pseudo-differential operator of order -1 with principal symbol $\frac{1}{2}\left(-\Delta_{\Gamma}\right)^{-1 / 2}$ (see [10]) and one takes the composition of the operators $\sqrt{-\Delta_{\Gamma}+1}$ and $\left(-\Delta_{\Gamma}\right)^{-1 / 2}$. Consequently, $\left(I d+P_{D}(\lambda)\right)^{-1}$ is a meromorphic operator-valued function and for $\operatorname{Re} \lambda<0$ one deduces

$$
\mathcal{N}(\lambda)=\left(I d+P_{D}(\lambda)\right)^{-1}\left(-\Delta_{\Gamma}+1\right)^{1 / 2}\left(I d-2 C_{00}(\lambda)\right)
$$


Since $\mathcal{N}(\lambda)$ is analytic for $\operatorname{Re} \lambda<0,-1$ is not an eigenvalue of $P_{D}(\lambda)$ for $\operatorname{Re} \lambda<0$. On the other hand, $C_{00}(\lambda)$ is a pseudo-differential operator of order -1 , hence it is compact one. The Neumann problem

$$
\left\{\begin{array}{l}
\left(-\Delta+\lambda^{2}\right) u=0 \text { in } \Omega, u \in H^{2}(\Omega), \\
\partial_{\nu} u=0 \text { on } \Gamma, \\
u:(\mathbf{i} \lambda)-\text { outgoing. }
\end{array}\right.
$$

has a non-trivial solution if the operator $2 C_{00}(\lambda)$ has eigenvalue 1 and this occurs only if $\lambda$ coincides with a resonance $\nu_{j}$, Re $\nu_{j}>0$, of the Neumann problem (see [6]). By Fredholm theorem one deduces that

$$
\mathcal{N}(\lambda)^{-1}=\left(I d-2 C_{00}(\lambda)\right)^{-1}\left(-\Delta_{\Gamma}+1\right)^{-1 / 2}\left(I d+P_{D}(\lambda)\right): H^{s}(\Gamma) \rightarrow H^{s+1}(\Gamma)
$$

is meromorphic with poles $\nu_{j}$.

Going back to the problem (1.2), for $\operatorname{Re} \lambda<0$ we write the boundary condition as follows

$$
\mathcal{C}(\lambda) v:=(\mathcal{N}(\lambda)-\lambda \gamma) v=\mathcal{N}(\lambda)\left(I d-\lambda \mathcal{N}(\lambda)^{-1} \gamma\right) v=0, v=f_{1} \in H^{1 / 2}(\Gamma)
$$

Clearly, for $\operatorname{Re} \lambda<0$ the operator $\mathcal{C}(\lambda)$ has the same singularities as $\mathcal{N}(\lambda)$, hence $\mathcal{C}(\lambda)$ : $H^{1 / 2}(\Gamma) \rightarrow H^{-1 / 2}(\Gamma)$ is analytic and satisfies the estimate (2.4) with another constant $A_{0}$. The operator $\mathcal{N}(\lambda)^{-1}$ is compact and by the results in [12] there are points $\lambda_{0}, \operatorname{Re} \lambda_{0}<0$, for which $I d-\lambda_{0} \mathcal{N}\left(\lambda_{0}\right)^{-1} \gamma$ is invertible. Applying the analytic Fredholm theorem for the operator $\left(I d-\lambda \mathcal{N}(\lambda)^{-1} \gamma\right)$ in the half planeRe $\lambda<0$, one concludes that

$$
\mathcal{C}(\lambda)^{-1}=\left(I d-\lambda \mathcal{N}(\lambda)^{-1} \gamma\right)^{-1} \mathcal{N}(\lambda)^{-1}: H^{-1 / 2}(\Gamma) \rightarrow H^{1 / 2}(\Gamma)
$$

is a meromorphic operator-valued function. Notice that for $\lambda \in \mathbb{R}^{-}$the operators $\mathcal{N}(\lambda), \mathcal{C}(\lambda)$ are self-adjoint. This follows from the Green formula for $\left(-\Delta+\lambda^{2}\right)$.

Remark 1. It is important to note that the analyticity of the resolvent $\left(-\Delta_{D}+\lambda^{2}\right)^{-1}$ for $\operatorname{Re} \lambda<0$ and the absence of resonances of the Neumann problem in the half plan $\{z \in \mathbb{C}:$ $\operatorname{Re} z<0\}$ imply that $\mathcal{C}(\lambda)^{-1}$ is meromorphic for $\operatorname{Re} \lambda<0$ and (2.5) is not necessary for the proof of this statement.

For the resolvent $(\lambda-G)^{-1}$ in [12] the following trace formula has been proved.

Proposition 1. Let $\delta \subset\{\lambda \in \mathbb{C}: \operatorname{Re} \lambda<0\}$ be a closed positively oriented curve without self intersections. Assume that $\mathcal{C}(\lambda)^{-1}$ has no poles on $\delta$. Then

$$
\operatorname{tr}_{\mathcal{H}} \frac{1}{2 \pi i} \int_{\delta}(\lambda-G)^{-1} d \lambda=\operatorname{tr}_{H^{1 / 2}(\Gamma)} \frac{1}{2 \pi i} \int_{\delta} \mathcal{C}(\lambda)^{-1} \frac{\partial \mathcal{C}}{\partial \lambda}(\lambda) d \lambda
$$

Since $G$ has only point spectrum in $\operatorname{Re} \lambda<0$, the left hand term in (2.8) is equal to the number of the eigenvalues of $G$ in the domain $\omega$ bounded by $\delta$ counted with their algebraic multiplicities. Setting $\tilde{\mathcal{C}}(\lambda)=\frac{\mathcal{N}(\lambda)}{\lambda}-\gamma$, we write the right hand side of (2.8) as

$$
\operatorname{tr} \frac{1}{2 \pi i} \int_{\delta} \tilde{\mathcal{C}}(\lambda)^{-1} \frac{\partial \tilde{\mathcal{C}}}{\partial \lambda}(\lambda) d \lambda
$$


Set $\lambda=-\frac{1}{\tilde{h}}, 0<\operatorname{Re} \tilde{h} \ll 1$ and consider the problem

$$
\left\{\begin{array}{l}
\left(-\tilde{h}^{2} \Delta+1\right) u=0 \text { in } \Omega \\
-\tilde{h} \partial_{\nu} u-\gamma u=0 \text { on } \Gamma \\
u-\text { outgoing. }
\end{array}\right.
$$

We introduce the operator $C(\tilde{h}):=-\tilde{h} \mathcal{N}\left(-\tilde{h}^{-1}\right)-\gamma$ and using (2.9), the trace formula (2.8) becomes

$$
\operatorname{tr} \frac{1}{2 \pi i} \int_{\delta}(\lambda-G)^{-1} d \lambda=\operatorname{tr} \frac{1}{2 \pi i} \int_{\tilde{\delta}} C(\tilde{h})^{-1} \dot{C}(\tilde{h}) d \tilde{h}
$$

where $\dot{C}$ denote the derivative with respect to $\tilde{h}$ and $\tilde{\delta}$ is the curve $\tilde{\delta}=\left\{z \in \mathbb{C}: z=-\frac{1}{w}, w \in\right.$ $\delta\}$.

Obviously, for $\lambda \in \Lambda$ one has $|\operatorname{Im} \lambda| \leq 1$ and this implies $\tilde{h} \in L$, where

$$
L:=\left\{\tilde{h} \in \mathbb{C}:|\operatorname{Im} \tilde{h}| \leq C_{1}|\tilde{h}|^{4},|\tilde{h}| \leq C_{0}^{-1}, \operatorname{Re} \tilde{h}>0\right\} .
$$

We write the points in $L$ as $\tilde{h}=h(1+\mathbf{i} \eta)$ with $0<h \leq h_{0} \leq C_{0}^{-1}, \eta \in \mathbb{R}$. Recall that $\frac{2 C_{1}}{C_{0}} \leq 1$. Then $\frac{C_{1}}{C_{0}^{3}} \leq 1 / 2$ and for $\tilde{h} \in L$ we get

$$
|\eta| \leq \frac{1}{2} \sqrt{1+\eta^{2}}
$$

hence $\eta^{2} \leq 1 / 3$. This implies

$$
|\eta| \leq C_{1} h\left(1+\eta^{2}\right)^{2} h^{2} \leq h^{2}, h(1+\mathbf{i} \eta) \in L,
$$

since $\frac{16 C_{1} h}{9} \leq 1$. Therefore the problem (2.10) becomes

$$
\left\{\begin{array}{l}
\left(-h^{2} \Delta-z\right) u=0 \text { in } \Omega, \\
-(1+\mathbf{i} \eta) h \partial_{\nu} u-\gamma u=0 \text { on } \Gamma, \\
u-\text { outgoing. }
\end{array}\right.
$$

with $z=-\frac{1}{(1+\mathbf{i} \eta)^{2}}=-1+s(\eta),|s(\eta)| \leq\left(2+h^{2}\right) h^{2} \leq 3 h^{2}$. On the other hand,

$$
C(\tilde{h})=-(1+\mathbf{i} \eta) h \mathcal{N}\left(-\tilde{h}^{-1}\right)-\gamma(x)
$$

\section{PARAmetrix FOr $N(h, z)$ in the Elliptic REgion}

In our exposition we will use $h$-pseudo-differential operators and we refer to [5] for more details. Let $X$ be a $C^{\infty}$ smooth compact manifold without boundary with dimension $d-1 \geq$ 1. Let $(x, \xi)$ be the coordinates in $T^{*}(X)$ and let $a(x, \xi ; h) \in C^{\infty}\left(T^{*}(X) \times\left(0, h_{0}\right]\right)$. Given $\ell, m \in \mathbb{R}$, one denotes by $S^{\ell, m}$ the set of symbols so that

$$
\left|\partial_{x}^{\alpha} \partial_{\xi}^{\beta} a(x, \xi ; h)\right| \leq C_{\alpha, \beta} h^{-\ell}(1+|\xi|)^{m-|\beta|}, \forall \alpha, \forall \beta, \quad(x, \xi) \in T^{*}(X) .
$$

If $\ell=0$, we denote $S^{\ell, m}$ by $S^{m}$. The $h$-pseudo-differential operator with symbol $a(x, \xi ; h)$ is defined by

$$
\left(O p_{h}(a) f\right)(x):=(2 \pi h)^{-d+1} \int_{T^{*} X} e^{\mathbf{i}\langle x-y, \xi\rangle / h} a(x, \xi ; h) f(y) d y d \xi
$$


We define the space of symbols $S_{c l}^{\ell, m}$ which have an asymptotic expansion

$$
a(x, \eta ; h) \sim \sum_{j=0}^{\infty} h^{j-\ell} a_{j}(x, \eta), a_{j} \in S^{m-j}
$$

and the corresponding classical pseudo-differential operator is given by

$$
(O p(a) f)(x):=(2 \pi)^{-d+1} \int_{T^{*} X} e^{\mathbf{i}\langle x-y, \eta\rangle} a(x, \eta ; h) f(y) d y d \eta .
$$

It is clear that by a change of variable $\xi=h \eta$ we may write a $h$ - pseudo-differential operator as a classical one with parameter $h$. We will use this fact in Section 4 . The operators with symbols in $S^{\ell, m}, S_{c l}^{\ell, m}$ are denoted by $L^{\ell, m}, L_{c l}^{\ell, m}$, respectively. The wave front $\widetilde{W F}(A) \subset \widetilde{T^{*}(\Gamma)}$ of an operator $A \in L^{\ell, m}$ is defined as in [15], where $\widetilde{T^{*}(\Gamma)}$ is the compactification of $T^{*}(\Gamma)$.

We will recall some results for the exterior semi-classical Dirichlet-to-Neumann map (see [16], [17], [12]). Consider the operator

$$
\mathcal{P}(h, z) u=\left(-h^{2} \Delta_{x}-z\right) u, z=-1+s(\eta) .
$$

In local normal geodesic coordinates $\left(y_{1}, y^{\prime}\right), y_{1}=\operatorname{dist}(y, \Gamma)$ in a neighbourhood $\mathcal{U}$ of $x_{0} \in \Gamma$ the operator $\mathcal{P}$ has the form (see [14])

$$
\mathcal{P}(h, z)=h^{2} D_{y_{1}}^{2}+r\left(y, h D_{y^{\prime}}\right)+h^{2} q(x) D_{y_{1}}-z, D_{j}=-\mathbf{i} \partial_{y_{j}}
$$

with $r\left(y, \eta^{\prime}\right)=\left\langle R(y) \eta^{\prime}, \eta^{\prime}\right\rangle, q(y) \in C^{\infty}$. Here

$$
R(y)=\left\{\sum_{k=1}^{d} \frac{\partial y_{m}}{\partial x_{k}} \frac{\partial y_{j}}{\partial x_{k}}\right\}_{m, j=2}^{d}=\left\{\left\langle\frac{\partial y_{m}}{\partial x}, \frac{\partial y_{j}}{\partial x}\right\rangle\right\}_{m, j=2}^{d}
$$

is a symmetric $((d-1) \times(d-1))$ matrix and $r\left(0, y^{\prime}, \eta^{\prime}\right)=r_{0}\left(y^{\prime}, \eta^{\prime}\right)$, where $r_{0}\left(y^{\prime}, \eta^{\prime}\right)$ is the principal symbol of the Laplace-Beltrami operator $-\Delta_{\Gamma}$ on $\Gamma$ equipped with the Riemannian metric induced by the Euclidean one in $\mathbb{R}^{d}$. For $z=-1+s(\eta)$ introduce $\rho\left(y^{\prime}, \eta^{\prime}, z\right)=$ $\sqrt{z-r_{0}\left(y^{\prime}, \eta^{\prime}\right)} \in C^{\infty}\left(T^{*} \Gamma\right)$ as the root of the equation

$$
\rho^{2}+r_{0}\left(y^{\prime}, \eta^{\prime}\right)-z=0
$$

with $\operatorname{Im} \rho\left(y^{\prime}, \eta^{\prime}, z\right)>0$. We have $\rho \in S^{1}$ and

$$
\sqrt{-1+s(\eta)-r_{0}}=\mathbf{i} \sqrt{1+r_{0}}-\frac{s(\eta)}{\sqrt{1-s(\eta)+r_{0}}+\mathbf{i} \sqrt{1+r_{0}}}
$$

which implies $\rho-\mathbf{i} \sqrt{1+r_{0}} \in S^{-1}$.

Let $u$ be the solution of the Dirichlet problem

$$
\left\{\begin{array}{l}
\left(-h^{2} \Delta-z\right) u=0 \text { in } \Omega, \\
u=f \text { on } \Gamma, \\
u-\text { outgoing. }
\end{array}\right.
$$

Consider the semi-classical Sobolev spaces $H_{h}^{k}(\Gamma)$ with norm $\left\|\left(1-h^{2} \Delta\right)^{s / 2} u\right\|_{L^{2}(\Gamma)}$ and introduce the exterior semi-classical Dirichlet-to-Neumann map

$$
N(h, z): H_{h}^{s}(\Gamma) \ni f \longrightarrow-\left.h \partial_{\nu} u\right|_{\Gamma} \in H_{h}^{s-1}(\Gamma) .
$$


G. Vodev [17] established for bounded domains $K \subset \mathbb{R}^{d}, d \geq 2$, with $C^{\infty}$ boundary and solutions $u$ of the Helmoltz equation $\left(-h^{2} \Delta-z\right) u=0, x \in K$, an approximation of the interior Dirichlet-to-Neumann map. With some modifications his results can be applied for the exterior Dirichlet-to-Neumann map $N(h, z)$ (see [12]). We need some information for the parametrix build in [17], [19] in the elliptic region $Z_{e}:=\{z \in \mathbb{C}: z=-1+s(\eta)\}$.

For the reader convenience we recall some points of the construction in [17], [19] for $z \in Z_{e}$. Let $\psi \in C_{0}^{\infty}\left(U_{0}\right), \psi=1$ in a neighbourhood $U_{0}$ of $x_{0} \in \Gamma$. Denote the local normal geodesic coordinates by $\left(x_{1}, x^{\prime}\right)$ and the dual variables by $\left(\xi_{1}, \xi^{\prime}\right)$. We search a parametrix $u_{\psi}$ of the problem (3.1) with boundary data $\psi f$ in the form

$$
\tilde{u}_{\psi}(x)=(2 \pi h)^{-d+1} \iint e^{\frac{i}{h}\left(\varphi\left(x, \xi^{\prime}, z\right)+\left\langle y^{\prime}, \xi^{\prime}\right\rangle\right)} \phi^{2}\left(\frac{x_{1}}{\delta}\right) a\left(x, \xi^{\prime}, h, z\right) f\left(y^{\prime}\right) d \xi^{\prime} d y^{\prime} .
$$

Here $0<\delta \ll 1$ and $\phi(t) \in C_{0}^{\infty}(\mathbb{R})$ is equal to 1 for $|t| \leq 1$ and to 0 for $|t| \geq 2$. We write

$$
R(x)=\sum_{k=0}^{N-1} x_{1}^{k} R_{k}\left(x^{\prime}\right)+x_{1}^{N} \mathcal{R}_{N}(x), q(x)=\sum_{k=0}^{N-1} x_{1} q_{k}\left(x^{\prime}\right)+x_{1}^{N} \mathcal{Q}_{N}(x) .
$$

For $\varphi$ the eikonal equation modulo $x_{1}^{N}$ becomes $\left(\partial_{x_{1}} \varphi\right)^{2}+\left\langle R(x) \partial_{x^{\prime}} \varphi, \partial_{x^{\prime}} \varphi\right\rangle-z=x_{1}^{N} \Phi_{N}$ and one obtains a smooth solution having the form

$$
\varphi=\sum_{k=0}^{N} x_{1}^{k} \varphi_{k}\left(x^{\prime}, \xi^{\prime}, z\right), \varphi_{0}=-\left\langle x^{\prime}, \xi^{\prime}\right\rangle,\left.\partial_{x_{1}} \varphi\right|_{x_{1}=0}=\varphi_{1}=\rho .
$$

The functions $\varphi_{k}$ satisfy for $0 \leq K \leq N-2$ the equalities

$$
\sum_{k+j=K}(k+1)(j+1) \varphi_{k+1} \varphi_{j+1}+\sum_{k+j+\ell=K}\left\langle R_{\ell} \nabla_{x^{\prime}} \varphi_{k}, \nabla_{x^{\prime}} \varphi_{j}\right\rangle-z=0 .
$$

Clearly, we can determine $\varphi_{K+1}$ from the above equality since $\rho \neq 0$. For $z=-1$ we have $\rho=\mathbf{i} \sqrt{1+r_{0}}$ and by recurrence one deduces $\varphi_{k}=\mathbf{i} \tilde{\varphi}_{k}$ with real-valued function $\tilde{\varphi}_{k}$. Thus for $z=-1$ we have $\varphi=-\left\langle x^{\prime}, \xi^{\prime}\right\rangle+\mathbf{i} \tilde{\varphi}$ with real-valued function $\tilde{\varphi}$. The amplitude of the parametrix has the form

$$
a=\sum_{j=0}^{N-1} h^{j} a_{j}\left(x, \xi^{\prime}, z\right),\left.a_{0}\right|_{x_{1}=0}=\psi,\left.a_{j}\right|_{x_{1}=0}=0, j \geq 1
$$

with $a_{j}=\sum_{k=0}^{N} x_{1}^{k} a_{k, j}\left(x^{\prime}, \xi^{\prime}, z\right), a_{0,0}=\psi, a_{0, j}=0, j \geq 1$. The functions $a_{j}$ satisfy the transport equations

$$
\begin{gathered}
2 \mathbf{i} \frac{\partial \varphi}{\partial x_{1}} \frac{\partial a_{j}}{\partial x_{1}}+2 \mathbf{i}\left\langle R(x) \nabla_{x^{\prime}} \varphi, \nabla_{x^{\prime}} a_{j}\right\rangle+\mathbf{i}(\Delta \varphi) a_{j}+\Delta a_{j-1} \\
=x_{1}^{N} A_{N}^{(j)}, 0 \leq j \leq N-1, a_{-1}=0
\end{gathered}
$$

We write (see Section 3 in [19])

$$
\Delta \varphi=\sum_{k=0}^{N-1} x_{1}^{k} \varphi_{k}^{\Delta}+x_{1}^{N} E_{N}(x), \Delta a_{j-1}=\sum_{k=0}^{N-1} x_{1}^{k} a_{k, j-1}^{\Delta}+x_{1}^{N} F_{N}^{(j-1)}(x)
$$

with

$$
\varphi_{k}^{\Delta}=(k+1)(k+2) \varphi_{k+2}+\sum_{\ell+\nu=k}\left(\left\langle R_{\ell} \nabla_{x^{\prime}}, \nabla_{x^{\prime}} \varphi_{\nu}\right\rangle+q_{\ell}(\nu+1) \varphi_{\nu+1}\right)
$$




$$
a_{k, j-1}^{\Delta}=(k+1)(k+2) a_{k+2, j-1}+\sum_{\ell+\nu=k}\left(\left\langle R_{\ell} \nabla_{x^{\prime}}, \nabla_{x^{\prime}} a_{\nu, j-1}\right\rangle+q_{\ell}(\nu+1) a_{\nu+1, j-1}\right) .
$$

This leads to the equality (see (3.18) in [19])

$$
\begin{aligned}
2 \mathbf{i} \sum_{k_{1}+k_{2}=k} & \left(k_{1}+1\right)\left(k_{2}+1\right) \varphi_{k_{1}+1} a_{k_{2}+1, j}+2 \mathbf{i} \sum_{k_{1}+k_{2}+k_{3}=k}\left\langle R_{k_{1}} \nabla_{x^{\prime}} \varphi_{k_{3}}, \nabla_{x^{\prime}} a_{k_{3}, j}\right\rangle \\
& +\sum_{k_{1}+k_{2}=k} \mathbf{i} \varphi_{k_{1}}^{\Delta} a_{k_{2}, j}=-a_{k, j-1}^{\Delta} \text { for } 0 \leq k \leq N-1,0 \leq j \leq N-1 .
\end{aligned}
$$

We can determine $a_{k, j}$ by recurrence from the above equality so that $a_{0,0}=\psi, a_{0, j}=0, j \geq$ $1, a_{k,-1}=0, k \geq 0$. Next introduce the operator

$$
T_{\psi}(h, z) f=-\left.h \frac{\partial \tilde{u}_{\psi}}{\partial x_{1}}\right|_{x_{1}=0}=O p_{h}\left(\tau_{\psi}\right) f
$$

with

$$
\tau_{\psi}=-\mathbf{i} \rho \psi-\sum_{j=0}^{N-1} h^{j+1} a_{1, j}, a_{1, j} \in S^{-j} .
$$

By using the outgoing resolvent $\left(h^{2} \Delta_{D}-z\right)^{-1}$ for the Dirichlet Laplacian in $\Omega$, we obtain a parametrix $u_{\psi}$ in $\Omega$ and for $z \in Z_{e}$ we have (see Prop. 2.2 in [12] and [17])

$$
\left\|\mathcal{N}(h, z)(\psi f)-T_{\psi} f\right\|_{H_{h}^{N}(\Gamma)} \leq C_{N} h^{-s_{d}+N}\|f\|_{L^{2}(\Gamma)}, \forall N \in \mathbb{N}
$$

with $C_{N}>0, s_{d}>0$ independent of $f, h$ and $z$ and $s_{d}$ independent of $N$. Taking a partition of unity $\sum_{j=1}^{M} \psi_{j}\left(x^{\prime}\right) \equiv 1$ on $\Gamma$, we construct a parametrix and define the operator $T(h, z)=\sum_{j=1}^{M} T_{\psi_{j}}(h, z)$. For $z=-1$ the symbol $\sqrt{1+r_{0}}+\sum_{j=0}^{N-1} h^{j+1} a_{1, j}$ of $T(h,-1)$ is real valued and we have the estimate (3.4) with $T_{\psi}(h, z)$ replaced by $T(h, z)$. Clearly, we may extend the symbol of $T(h, z)$ holomorphically for $\tilde{h} \in L$.

\section{Properties of the operator $P(h)$}

In this section we assume that $\gamma(x)>1, \forall x \in \Gamma$ and we study the operator $P(h)=$ $T(h,-1)-\gamma(x)$ when $h$ is real. Set

$$
\min _{x \in \Gamma} \gamma(x)=c_{0}>1, \max _{x \in \Gamma} \gamma(x)=c_{1} \geq c_{0}
$$

and choose a constant $C=\frac{2}{c_{1}^{2}}$. As we mentioned in Section 3, we can consider the operator $P(h)$ as a classical pseudo-differential operator $O p(P)$ with parameter $h$ with classical symbol $P=\sqrt{1+h^{2} r_{0}}-\gamma+h P_{0}(x, h \xi), P_{0}(x, \xi) \in S^{0}$. We denote by $(.,$.$) the scalar product in$ $L^{2}(\Gamma)$ and for two self adjoint operators $L_{1}, L_{2}$ the inequality $L_{1} \geq L_{2}$ means $\left(L_{1} u, u\right) \geq$ $\left(L_{2} u, u\right), \forall u \in L^{2}(\Gamma)$.

Proposition 2. Let $\langle h \Delta\rangle=\left(1-h^{2} \Delta_{\Gamma}\right)^{1 / 2}$ and let $\epsilon=C\left(c_{0}-1\right)^{2}<2$. Then for $h$ sufficiently small we have

$$
h \frac{\partial P(h)}{\partial h}+C P(h)\langle h \Delta\rangle^{-1 / 2} P(h) \geq \epsilon\left(1-C_{2} h\right)\langle h \Delta\rangle
$$

with a constant $C_{2}>0$ independent of $h$. 
Proof. The principal symbol of the operator on the left hand side in (4.1) has the form

$$
\begin{array}{r}
q_{1}=2 h^{2} r_{0}\left(1+h^{2} r_{0}\right)^{-1 / 2}+C \sqrt{1+h^{2} r_{0}}-2 C \gamma(x)+C \gamma^{2}(x)\left(1+h^{2} r_{0}\right)^{-1 / 2} \\
=(2+C-\epsilon) \sqrt{1+h^{2} r_{0}}-2 C \gamma(x)+\left(C \gamma^{2}(x)-2\right)\left(1+h^{2} r_{0}\right)^{-1 / 2} \\
+\epsilon \sqrt{1+h^{2} r_{0}}
\end{array}
$$

Clearly,

$$
((2+C-\epsilon)\langle h D\rangle u, u) \geq((2+C-\epsilon) u, u)
$$

and

$$
C \gamma^{2}(x)-2 \leq C c_{1}^{2}-2=0
$$

Therefore,

$$
\begin{gathered}
\left(\left(C \gamma^{2}(x)-2\right)\langle h D\rangle^{-1 / 2} u, u\right)=\left(\left(C \gamma^{2}(x)-2\right)\left(\langle h D\rangle^{-1 / 2}-1\right) u, u\right) \\
+\left(\left(C \gamma^{2}(x)-2\right) u, u\right) \geq\left(\left(C \gamma^{2}(x)-2\right) u, u\right)-h C_{1}\|u\|^{2}, 0<h \leq h_{0} .
\end{gathered}
$$

Here the operator $\left(C \gamma^{2}(x)-2\right)\left(\langle h D\rangle^{-1 / 2}-1\right)$ has non-negative (classical) principal symbol

$$
\frac{\left(2-C \gamma^{2}(x)\right) h^{2} r_{0}}{1+h^{2} r_{0}+\sqrt{1+h^{2} r_{0}}}
$$

and applied the semi-classical sharp Gärding inequality (see for instance, [5], Theorem 7.12). Taking into account (4.2) and the inequality $C(\gamma(x)-1)^{2}-\epsilon \geq C\left(c_{0}-1\right)^{2}-\epsilon=0$, one deduces

$$
\begin{gathered}
\left.\left(O p\left(q_{1}\right) u, u\right) \geq\left(\left(C(\gamma(x)-1)^{2}\right)-\epsilon\right) u, u\right)+\epsilon(\langle h D\rangle u, u)-h C_{1}\|u\|^{2} \\
\geq \epsilon(\langle h D\rangle u, u)-h C_{1} h\|u\|^{2} .
\end{gathered}
$$

The full symbol of the operator on the right hand side of (4.1) has the form $q_{1}+h q_{0}$. The term $h\left(O p\left(q_{0}\right) u, u\right)-h C_{1}\|u\|^{2}$ can be absorbed by $\epsilon C_{2} h(\langle h D\rangle u, u)$ taking $\epsilon C_{2} \geq C_{1}+$ $\left\|O p\left(q_{0}\right)\right\|_{L^{2} \rightarrow L^{2}}$ and this completes the proof.

Remark 2. The values of $\epsilon$ depends on $\left(c_{0}-1\right)^{2}$ and $\epsilon \searrow 0$ as $c_{0} \searrow 1$. In the case when $\gamma \equiv$ const and $K$ is the ball $\{x:\|x\| \leq 1\}$ the operator $G$ has no eigenvalues if $\gamma \equiv 1$ (see [12]). Moreover, in this case for $\gamma>1$ the eigenvalues of $G$ lie in the interval $\left(-\infty,-\frac{1}{\gamma-1}\right)$. Thus as $\gamma \searrow 1$, in the domain $\operatorname{Re} \lambda>-\frac{1}{\gamma-1}$ there are no eigenvalues.

Next we follow the argument of Section 4, [15] with some modifications. Consider the semi-classical Sobolev space $H^{s}(\Gamma)$ with norm $\|u\|_{s}=\left\|\langle h D\rangle^{s} u\right\|_{L^{2}}$. The operator $P(h)$ : $H^{1} \rightarrow L^{2}$ has derivative $\dot{P}(h)=\mathcal{O}\left(h^{-1}\right): H^{1} \rightarrow L^{2}$. Denote by

$$
\mu_{1}(h) \leq \mu_{2}(h) \leq \ldots \leq \mu_{k}(h) \leq \ldots
$$

the eigenvalues of $P(h)$ repeated with their multiplicities.

Let $h_{1}$ be small and let $\mu_{k}\left(h_{1}\right)$ have multiplicity $m$. For $h$ close to $h_{1}$ one has exactly $m$ eigenvalues and we denote by $F(h)$ the space spanned by them. We can find a small interval $(\alpha, \beta)$ around $\mu_{k}\left(h_{1}\right)$, independent on $h$, containing the eigenvalues spanning $F(h)$. Given $h_{2}>h_{1}$ close to $h_{1}$, consider a normalised eigenfunction $e\left(h_{2}\right)$ with eigenvalue $\mu_{k}\left(h_{2}\right)$. Let $\pi(h)=E_{(\alpha, \beta)}$ be the spectral projection of $P(h)$, hence $F(h)=\pi(h) L^{2}(\Gamma)$. Then $(\pi(h)-$ $I) \pi(h)=0$ yields $\pi(h) \dot{\pi}(h) \pi(h)=0$ and we deduce $\left.\dot{\pi}(h)\right|_{F(h)}=0$. We construct a smooth extension $e(h) \in F(h), h \in\left[h_{1}, h_{2}\right]$ of $e\left(h_{2}\right)$ with $\|e(h)\|=1, \dot{e}(h) \in F(h)^{\perp}$. Obviously, $e\left(h_{1}\right)$ will be normalised eigenfunction with eigenvalue $\mu_{k}\left(h_{1}\right)$. 
Considering the eigenvalues $\mu_{k}(h)$ of $P(h)$ in a small interval $[-\delta, \delta], \delta>0$, one gets $\|P(h) e(h)\| \leq \delta$. On the other hand,

$$
h \dot{P}(h)=h^{2} \Delta\langle h D\rangle^{-1}+h L_{0}=P(h)-\langle h D\rangle^{-1}+h L_{1}
$$

with zero order operators $L_{0}, L_{1}$ and this implies $|(\dot{P}(h) e(h), e(h))| \leq C_{0} h^{-1}, h \in\left[h_{1}, h_{2}\right]$. Therefore

$$
\left|\mu_{k}\left(h_{2}\right)-\mu_{k}\left(h_{1}\right)\right|=\left|\int_{h_{1}}^{h_{2}} \frac{d}{d h}(P(h) e(h), e(h)) d h\right| \leq C_{0} \int_{h_{1}}^{h_{2}} h^{-1} d h \leq \frac{C_{0}}{h_{1}}\left(h_{2}-h_{1}\right) .
$$

Assuming $\mu_{k}(h) \in[-\delta, \delta]$, we deduce that $\mu_{k}(h)$ is locally Lipschitz function in $h$ and its almost defined derivative satisfies $\left|\frac{\partial \mu_{k}(h)}{\partial h}\right| \leq C_{0} h^{-1}$.

To estimate $h \frac{\partial \mu_{k}(h)}{\partial h}$ from below, we exploit Proposition 2 and apply (4.1). For $h \leq h_{0} \leq$ $\frac{1}{8 C_{2}}$ and $\mu_{k}(h) \in[-\delta, \delta]$ we have

$$
\begin{aligned}
h \frac{\partial \mu_{k}(h)}{\partial h}=(h \dot{P}(h) e(h), e(h)) \geq & \epsilon\left(1-C_{2} h\right)(\langle h D\rangle e(h), e(h))-C\left(\langle h D\rangle^{-1} P(h) e(h), P(h) e(h)\right) \\
& \geq \epsilon\left(1-C_{2} h\right)-C \delta^{2} \geq \frac{3 \epsilon}{4}
\end{aligned}
$$

choosing

$$
\delta=\left(c_{0}-1\right) \sqrt{\frac{1}{4}-C_{2} h_{0}} \geq \frac{\left(c_{0}-1\right)}{2 \sqrt{2}} .
$$

Consequently, for $h \in\left[h_{1}, h_{2}\right]$ one has

$$
\mu_{k}\left(h_{2}\right)-\mu_{k}\left(h_{1}\right) \geq \frac{3 \epsilon}{4} \int_{h_{1}}^{h_{2}} h^{-1} d h \geq \frac{3 \epsilon}{4 h_{2}}\left(h_{2}-h_{1}\right)
$$

and we obtain

$$
\frac{3 \epsilon}{4} \leq h \frac{d \mu_{k}(h)}{d h} \leq C_{0}
$$

Fixing $h_{0}>$ small, we conclude that the eigenvalue $\mu_{k}(h)$ increases when $h$ increases and $\mu_{k}(h) \in[-\delta, \delta]$. It is well known (see for instance, [5]) that

$$
\sharp\left\{k: \mu_{k}\left(h_{0}\right) \leq 0\right\}=\kappa_{0}=\frac{1}{\left(2 \pi h_{0}\right)^{d-1}} \int_{p_{1}(x, \xi) \leq 0} d x d \xi+\mathcal{O}\left(h_{0}^{-d+2}\right),
$$

$p_{1}(x, \xi)$ being the principal symbol of $P(\operatorname{Re} h)$. Then for $k>\kappa_{0}$ we have $\mu_{k}\left(h_{0}\right)>0$ and if for $h<h_{0}$ one has $\mu_{k}(h)<0$, then there exists a point $h<h_{k}<h_{0}$ with the properties $\mu_{k}\left(h_{k}\right)=0, \mu_{k}(h)<0$ for $0<h<h_{k}$. This implies that there exists a sequence $h_{k_{0}} \geq h_{k_{0}+1} \geq \ldots$ of values $0<h \leq h_{0}$ such that $\mu_{k}\left(h_{k}\right)=0, k_{0}>\kappa_{0}$. These values $h_{k}$ are precisely those for which $P(h)$ is not invertible. Next we choose $p>d$ and construct the intervals $I_{k, p}$ containing $h_{k}$ with length $\left|I_{k, p}\right| \sim h^{p+1}$ and $\left|\mu_{k}(h)\right| \geq h^{p}$ for $h \in\left(0, h_{0}\right] \backslash I_{k, p}$. As in [15], one constructs the disjoint intervals $J_{k, p}$, and we obtain the following

Proposition 3 (Prop. 4.1, [15]). Let $p>d$ be fixed. The inverse operator $P(h)^{-1}: L^{2} \rightarrow L^{2}$ exists and has norm $\mathcal{O}\left(h^{-p}\right)$ for $h \in\left(0, h_{0}\right] \backslash \Omega_{p}$, where $\Omega_{p}$ is a union of disjoint closed intervals $J_{1, p}, J_{2, p}, \ldots$ with $\left|J_{k, p}\right|=\mathcal{O}\left(h^{p+2-d}\right)$ for $h \in J_{k, p}$. Moreover, the number of such intervals that intersect $[h / 2, h]$ for $0<h \leq h_{0}$ is at most $\mathcal{O}\left(h^{1-p}\right)$. 


\section{Relations Between the trace integrals for $C(h)$ and $P(h)$}

In this section we study the operators $C(h)$ and $P(h)$ for complex $h \in L$. We use the notation $h$ instead of $\tilde{h}$ used in Sections 2, 3. For $z=-1$ the operator $T(\operatorname{Re} h,-1)$ constructed in Section 3 has principal semi-classical symbol $\sqrt{1+r_{0}}$, so it is elliptic. The ellipticity holds also for the operator $T(h, z), h \in L, z=-1+s(\eta)$, holomorphic with respect to $h$, provided $|h|$ small enough. On the other hand, $P(h)=(1+\mathbf{i} \eta) T(h, z)-\gamma(x)$ is not elliptic and for $h \in \mathbb{R}, \eta=0, z=-1$ its semi-classical principal symbol vanishes on the set

$$
\Sigma=\left\{(x, \xi) \in T^{*}(\Gamma): r_{0}(x, \xi)=\gamma^{2}-1\right\} .
$$

For the symbol $r_{0}(x, \xi)$ of the Laplace-Beltrami operator on $\Gamma$ there exists a constant $C_{3}>0$ such that $r_{0}(x, \xi) \geq C_{3}\|\xi\|^{2},(x, \xi) \in T^{*}(\Gamma)$. Choose a constant $B_{0}>0$ so that $\sqrt{C_{3}} B_{0} \geq 2 c_{1}$ and consider a symbol $\chi(x, \xi) \in C_{0}^{\infty}\left(T^{*}(\Gamma)\right), 0 \leq \chi(x, \xi) \leq 2$ such that

$$
\chi(x, \xi)= \begin{cases}2, & x \in \Gamma,\|\xi\| \leq B_{0} \\ 0, & x \in \Gamma,\|\xi\| \geq B_{0}+1 .\end{cases}
$$

Introduce the operator

$$
\tilde{M}(h)=P(\operatorname{Re} h)+\gamma(x) \chi\left(x, h D_{x}\right)=T(\operatorname{Re} h,-1)+\gamma(x)\left(\chi\left(x, h D_{x}\right)-1\right) .
$$

The principal symbol of $\tilde{M}(h)$ has the form

$$
\tilde{m}(x, \xi)=\sqrt{1+r_{0}}+\gamma(x)(\chi(x, \xi)-1) .
$$

Clearly, $\tilde{M}(h)$ is elliptic since for $\|\xi\| \leq B_{0}$ one gets $\operatorname{Re} \tilde{m}(x, \xi) \geq c_{0}$, while for $\|\xi\|>B_{0}$ we have

$$
|\tilde{m}(x, \xi)| \geq \sqrt{C_{3}}\|\xi\|-c_{1} \geq \frac{\sqrt{C_{3}}}{2}\|\xi\|+\frac{\sqrt{C_{3}}}{2} B_{0}-c_{1} \geq \frac{\sqrt{C_{3}}}{2}\|\xi\| .
$$

Consequently, $\tilde{m}(x, \xi) \in S_{0}^{1}$, the operator $\tilde{M}(h)^{-1}: H^{s}-H^{s+1}$ is bounded by $\mathcal{O}_{s}(1)$ and $\widetilde{W F}(P(\operatorname{Re} h)-\tilde{M}(h)) \cap\left\{\|\xi\| \gg B_{0}+1\right\}=\emptyset$. Since $\chi(x, \xi)$ vanishes for $\|\xi\| \geq B_{0}+1$, by applying Proposition A.1 in [15], we can extend holomorphically $\chi\left(x, h D_{x}\right)$ to $\eta\left(x, \tilde{h} D_{x}\right)$ in the domain $L$. As we mentioned in Section 3, the operator $P(h)$ also has a holomorphic extension for $\tilde{h} \in L$. Thus $\tilde{M}(h)$ has a holomorphic extension

$$
M(h)=P(h)+\gamma(x)\left(\eta\left(x, \tilde{h} D_{x}\right)-1\right)
$$

for $\tilde{h} \in L$ and $\widetilde{W F}(P(h)-M(h)) \cap\left\{\|\xi\| \gg B_{0}+1\right\}=\emptyset$. The last relation implies $P(h)-$ $M(h): \mathcal{O}(1): H^{-s} \rightarrow H^{s}, \forall s$.

Now we can repeat without any change the proof of Lemma 5.1 in [15], exploiting Proposition 2. First we obtain

$$
\left\|P(h)^{-1}\right\|_{\mathcal{L}\left(H^{-1 / 2}, H^{1 / 2}\right)} \leq C \frac{\operatorname{Re} h}{|\operatorname{Im} h|}, \operatorname{Re} h>0, \operatorname{Im} h \neq 0 .
$$

Next, one deduces the estimate

$$
\left\|P(h)^{-1}\right\|_{\mathcal{L}\left(H^{s}, H^{s+1}\right)} \leq C_{s} \frac{\operatorname{Re} h}{|\operatorname{Im} h|}, \operatorname{Re} h>0, \operatorname{Im} h \neq 0
$$

applying (5.1) and the representation

$$
P^{-1}=M^{-1}-M^{-1}(P-M) M^{-1}+M^{-1}(P-M) P^{-1}(P-M) M^{-1},
$$


combined with the property of $P(h)-M(h)$ mentioned above. Following [15], introduce a piecewise smooth simply positively oriented curve $\gamma_{k . p}$ as a union of four segments: Re $h \in$ $J_{k, p}, \operatorname{Im} h= \pm(\operatorname{Re} h)^{p+1}$ and $\operatorname{Re} h \in \partial J_{k, p},|\operatorname{Im} h| \leq(\operatorname{Re} h)^{p+1}$, where $J_{k, p}$ is one of the intervals in $\Omega_{p}$ defined in Proposition 3. Then we have

Proposition 4 (Prop. 5.2, [15]). For every $h \in \gamma_{k, p}$ the inverse operator $P(h)^{-1}$ exists and

$$
\left\|P(h)^{-1}\right\|_{\mathcal{L}\left(H^{s}, H^{s+1}\right)} \leq C_{s}(\operatorname{Re} h)^{-p}, h \in \gamma_{k, p}
$$

To estimate $C(h)^{-1}$, we write

$$
\begin{gathered}
C(h)=-(1+\mathbf{i} \eta) h N(\operatorname{Re} h, z)-\gamma(x)=(1+\mathbf{i} \eta) T(\operatorname{Re} h, z)-\gamma(x)+\mathcal{R}_{m}(\operatorname{Re} h, z) \\
=P(h)+\mathcal{R}_{m}(h, z), m \gg 2 p
\end{gathered}
$$

with $\mathcal{R}_{m}(h, z): \mathcal{O}\left((\operatorname{Re} h)^{m}\right): H^{s} \rightarrow H^{s+m-1}$. Therefore

$$
C(h) P(h)^{-1}=I d+\mathcal{R}_{m}(\operatorname{Re} h, z) P(h)^{-1}
$$

and Proposition 4 imply

$$
\left\|\mathcal{R}_{m}(\operatorname{Re} h, z) P(h)^{-1}\right\|_{\mathcal{L}\left(H^{s}, H^{s+m}\right)} \leq C_{s}(\operatorname{Re} h)^{-p+m} .
$$

For small Re $h$ the operator on the right hand side of (5.3) is invertible and

$$
C(h) P(h)^{-1}\left(I d+\mathcal{R}_{m}(\operatorname{Re} h, z) P(h)^{-1}\right)^{-1}=I d .
$$

On the other hand, the operator $C(h)$ is elliptic for $|\xi| \gg 1$ and this implies that $C(h)$ : $H^{1 / 2} \rightarrow H^{-1 / 2}$ is a Fredholm operator. The index of $C(h)$ is constant for $h \in L$ and according to the results in [12], this index is 0 . Hence the right inverse to $C(h)$ is also a left inverse, so it is two side inverse. Thus we obtain

$$
\left\|C(h)^{-1}\right\|_{\mathcal{L}\left(H^{s}, H^{s+1}\right)} \leq C_{s}(\operatorname{Re} h)^{-p}, h \in \gamma_{k, p}
$$

Moreover,

$$
C(h)^{-1}-P(h)^{-1}=P(h)^{-1}\left(\left(I d+\mathcal{R}_{m}(\operatorname{Re} h, z) P(h)^{-1}\right)^{-1}-I d\right)=K(h)
$$

with $K(h)=\mathcal{O}_{s}\left(|h|^{m-2 p}\right): H^{s} \rightarrow H^{s+m+1}, \forall s, h \in \gamma_{k, p}$. To estimate $\dot{C}(h)-\dot{P}(h)$, notice that $C(h)-P(h)$ is holomorphic with respect to $h$ in $L_{0}$ and by Cauchy formula

$$
\dot{C}(h)-\dot{P}(h)=\frac{1}{2 \pi \mathbf{i}} \int_{\tilde{\gamma}_{k, p}} \frac{C(\zeta)-P(\zeta)}{\zeta-h} d \zeta=\frac{1}{2 \pi \mathbf{i}} \int_{\tilde{\gamma}_{k, p}} \frac{\mathcal{R}_{m}(\operatorname{Re} h, z)}{\zeta-h} d \zeta=K^{\prime}(h),
$$

where $\tilde{\gamma}_{k, p}$ is the boundary of a domain containing $\gamma_{k, p}$ with the property dist $\left(\tilde{\gamma}_{k, p}, \gamma_{k . p}\right) \geq$ $(\operatorname{Re} h)^{p}$. Thus yileds

$$
K^{\prime}(h)=\mathcal{O}_{s}\left(|h|^{m-p}\right): H^{s} \rightarrow H^{s+m+1}, \forall s, h \in \gamma_{k, p}
$$

Concerning the operator $P(h)$, we obtain a trace formula repeating without any change the argument in [15]. Let $\mu_{k}\left(h_{k}\right)=0, k \geq k_{0}$. It is easy to see that $\mu_{k}(h)$ has no other zeros for $0<h \leq h_{0}$, exploiting the fact that $\mu_{k}(h)$ in increasing for $\mu_{k}(h) \in[-\delta, \delta]$. One defines the multiplicity of $h_{k}$ as the multiplicity of the eigenvalue $\mu_{k}\left(h_{k}\right)$. Then we have 
Proposition 5 (Prop. 5.3, [15]). Let $\beta \subset L$ be a closed positively oriented $C^{1}$ curve without self intersections which avoids the points $h_{k}$ with $\mu_{k}\left(h_{k}\right)=0$. Then

$$
\operatorname{tr} \frac{1}{2 \pi \mathbf{i}} \int_{\beta} P(h)^{-1} \dot{P}(h) d h
$$

is equal to the number of $h_{k}$ in the domain bounded by $\beta$.

Now we may compare the trace formula for $C(h)$ and $P(h)$. First we compare the integrals over $\gamma_{k, p}$. We have

$$
\begin{gathered}
\operatorname{tr} \frac{1}{2 \pi \mathbf{i}} \int_{\gamma_{k, p}} C(h)^{-1} \dot{C}(h) d h=\operatorname{tr} \frac{1}{2 \pi \mathbf{i}} \int_{\gamma_{k, p}}\left(C(h)^{-1}-P(h)^{-1}\right) \dot{C}(h) d h \\
+\operatorname{tr} \frac{1}{2 \pi \mathbf{i}} \int_{\gamma_{k, p}} P(h)^{-1} \dot{C}(h) d h=\operatorname{tr} \frac{1}{2 \pi \mathbf{i}} \int_{\gamma_{k, p}} P(h)^{-1} \dot{C}(h) d h+\mathcal{O}_{p}\left((\operatorname{Re} h)^{m-2 p}\right) .
\end{gathered}
$$

Here we have used (5.5) and the estimate

$$
\|\dot{C}(h)\|_{\mathcal{L}\left(H^{1 / 2}, H^{-1 / 2}\right)} \leq C|h|^{-2}, h \in L .
$$

which follows from (2.4). Next the property of $K^{\prime}(h)$ yields

$$
\operatorname{tr} \frac{1}{2 \pi \mathbf{i}} \int_{\gamma_{k, p}} P(h)^{-1} \dot{C}(h) d h=\operatorname{tr} \frac{1}{2 \pi \mathbf{i}} \int_{\gamma_{k, p}} P(h)^{-1} \dot{P}(h) d h+\mathcal{O}_{p}\left((\operatorname{Re} h)^{m-2 p}\right) .
$$

For small $h$ and $m \gg 2 p$ the terms $\mathcal{O}_{p}\left((\operatorname{Re} h)^{m-2 p}\right)$ are negligible and we obtain, as in [15], a map $\ell_{p}$ between the set of points $h_{k} \in(0, h(p)$ ] counted with their multiplicities and the eigenvalues $\ell_{p}\left(h_{k}\right) \in \Lambda$ counted with their multiplicities. The number of points $h_{k} \in J_{k, p}$ counted with their multiplicities is equal to the number of eigenvalues $\lambda_{j}=\ell\left(h_{k}\right)$ of $G$ counted with their multiplicities lying in $\Lambda_{k, p}=\left\{z \in \mathbb{C}: z=-\frac{1}{\zeta}, \zeta \in \omega_{k, p}\right\}, \omega_{k, p} \subset L$ being the domain bounded by $\gamma_{k, p}$. Notice that for a point $h_{k}$ we could have many $\lambda_{j} \in \ell_{p}\left(h_{k}\right) \subset \Lambda_{k, p}$. On the other hand, for every $\lambda_{j} \in \ell_{p}\left(h_{k}\right)$ one has

$$
\left|\lambda_{j}+\frac{1}{h_{k}}\right| \leq C_{p} h_{k}^{p+2-d} .
$$

The integral over $\beta$ in Proposition 5 can be presented as a sum of integrals over $\gamma_{k, p}$ plus integrals over curves $\alpha_{j, p}$ which are the boundary of domains $\beta_{j, p}$ such that $\beta_{j, p} \cap \Omega_{p}=\emptyset, \forall j$. By Proposition 3 for $h \in\left(0, h_{0}\right] \backslash \Omega_{p}$ the operator $P(h)$ is invertible. Applying an argument similar to that used above, one concludes that $P(h)$ is invertible for $h \in \beta_{j, p}$. Consequently, there are no contributions from the integrals over $\alpha_{j, p}$ and we must sum the contributions over the integrals over $\gamma_{k, p}$, that is the sum of the number of the corresponding points $h_{k}$.

Consider the counting function

$$
\mathbf{N}(r)=\sharp\left\{\lambda \in \sigma_{p}(G) \cap \Lambda:|\lambda| \leq r, \operatorname{Re} \lambda \leq-C_{0}\right\}, r>C_{0}
$$

with $h_{0}^{-1}=C_{0}>0$ large enough. Then for $|\lambda| \leq r$ we must consider $r^{-1}<|\tilde{h}|, \tilde{h} \in L$. Modulo a finite number eigenvalues (see Section 4 and the number $\kappa_{0}$ ), we are going to count the points $r^{-1}<h_{k} \leq h_{0}$ and the number of the eigenvalues $\mu_{k}(h)$ for which we have $\mu_{k}\left(h_{k}\right)=0$. Hence we have $\mu_{k}\left(r^{-1}\right)<0$, since otherwise we obtain a contradiction. The 
problem is reduced to find the number of the negative eigenvalues of $P\left(r^{-1}\right)$ which is given by well known formula

Clearly,

$$
\frac{r^{d-1}}{(2 \pi)^{d-1}} \int_{p_{1}(x, \xi) \leq 0} d x d \xi+\mathcal{O}_{\gamma}\left(r^{d-2}\right)
$$

$$
\int_{p_{1}(x, \xi) \leq 0} d x d \xi=\int_{r_{0}(x, \xi) \leq \gamma^{2}(x)-1} d x d \xi=\int_{\Gamma}\left(\gamma^{2}(x)-1\right)^{(d-1) / 2}\left(\int_{r_{0}(x, \eta) \leq 1} d \eta\right) d x .
$$

For the induced Riemannian metric on $\Gamma$ the integral over the dual variable $\eta$ yields the volume $\omega_{d-1}$ of the unit ball $\left\{x \in \mathbb{R}^{d-1}:|x| \leq 1\right\}$ and we obtain the asymptotic (1.5). This completes the proof of Theorem 1.

\section{Generalisations}

We may study with some modifications a more general dissipative boundary problem

$$
\left\{\begin{array}{l}
u_{t t}-\Delta_{x} u+c(x) u_{t}=0 \text { in } \mathbb{R}_{t}^{+} \times \Omega, \\
\partial_{\nu} u-\gamma(x) \partial_{t} u-\sigma(x) u=0 \text { on } \mathbb{R}_{t}^{+} \times \Gamma, \\
u(0, x)=f_{1}, u_{t}(0, x)=f_{2},
\end{array}\right.
$$

where $c(x) \geq 0, \sigma(x) \geq 0$ are smooth functions defined respectively in $\mathbb{R}^{d}$ and $\Gamma$ and $c(x)=0$ for $|x| \geq R_{0}>0$ (see [8]). The solution is given by a semi-group $V(t)=e^{t G}, t \geq 0$ with $f=\left(f_{1}, f_{2}\right)$ in the energy space $\mathcal{H}_{E}$ with norm

$$
\|f\|_{\mathcal{H}_{E}}^{2}=\int_{\Omega}\left(\left|\nabla_{x} f_{1}\right|^{2}+\left|f_{2}\right|^{2}\right) d x+\int_{\Gamma} \sigma\left|f_{1}\right|^{2} d y
$$

The generator of $V(t)$ has the form

$$
G=\left(\begin{array}{ll}
0 & 1 \\
\Delta & c
\end{array}\right)
$$

with a domain $D(G)$ being the closure in the graph norm

$$
\|\| f \|\left.\right|_{E}=\left(\|f\|_{\mathcal{H}_{E}}^{2}+\|G f\|_{\mathcal{H}_{E}}^{2}\right)^{1 / 2}
$$

of functions $f=\left(f_{1}, f_{2}\right) \in C_{(0)}^{\infty}\left(\mathbb{R}^{d}\right) \times C_{(0)}^{\infty}\left(\mathbb{R}^{d}\right)$ satisfying the boundary condition $\partial_{\nu} f_{1}-$ $\gamma f_{2}-\sigma f_{1}=0$ on $\Gamma$. If we have an eigenfunction $f=\left(f_{1}, f_{2}\right)$ with $G f=\lambda f$, and $\lambda=-\frac{1}{\tilde{h}}$ (for simplicity we keep the notation of Section 2), then $u=f_{1}$ is a solution of the problem

$$
\left\{\begin{array}{l}
\left(-\tilde{h}^{2} \Delta+1-\tilde{h} c\right) u=0 \text { in } \Omega \\
-\tilde{h} \partial_{\nu} u-\gamma u+\tilde{h} \sigma u=0 \text { on } \Gamma \\
u-\text { outgoing. }
\end{array}\right.
$$

Therefore with $\tilde{h}=h(1+\mathbf{i} \eta), \eta \in \mathbb{R}, z=-\frac{1}{(1+\mathbf{i} \eta)^{2}}$ we obtain the problem

$$
\left\{\begin{array}{l}
\left(-h^{2} \Delta-z-\frac{h}{1+\mathbf{i} \eta} c\right) u=0 \text { in } \Omega, \\
-(1+\mathbf{i} \eta) h \partial_{\nu} u-\gamma u+h(1+\mathbf{i} \eta) \sigma u=0 \text { on } \Gamma, \\
u-\text { outgoing. }
\end{array}\right.
$$

We need to consider the semi-classical exterior Dirichlet-to-Neumann operator $N(h, z)$ related to the operator $-h^{2} \Delta-z-\frac{h}{1+\mathbf{i} \eta} c$. The construction of the semi-classical paramterix for 
$N(h, z)$ is the same as in [17], [12]. The term $\frac{h}{1+\mathbf{i} \eta} c$ is lower order operator and the principal symbol of $N(h, z)$ is $\sqrt{1+r_{0}}$. Next we deal with the operator

$$
P(\tilde{h})=(1+\mathbf{i} \eta) N(h, z)-\gamma-h(1+\mathbf{i} \eta) \sigma
$$

and the self-adjoint operator $P(h)=N(h, z)-\gamma-h \sigma$. Hear $h(1+\mathbf{i} \eta) \sigma$ is a lower order operator and we may repeat the arguments of Sections 4, 5. Under the assumptions of Theorem 1 one obtains a Weyl formula (1.5) with the same leading term. We leave the details to the reader.

We hope that our arguments combined with the construction of a semi-classical parametrix in [20] can be applied for the analysis of the eigenvalues of Maxwell's equations with dissipative boundary conditions

$$
\left\{\begin{array}{l}
\partial_{t} E-\operatorname{curl} H=0, \partial_{t} H+\operatorname{curl} E=0 \text { in } \mathbb{R}_{t}^{+} \times \Omega \\
\nu \wedge E-\gamma(x)(\nu \wedge \nu \wedge H)=0 \text { on } \mathbb{R}_{t}^{+} \times \Gamma \\
E(0, x)=E_{0}(x), H(0, x)=H_{0}(x)
\end{array}\right.
$$

where $d=3,\left(E_{0}, H_{0}\right) \in L^{2}\left(\mathbb{R}_{t}^{+} \times \Omega: \mathbb{C}^{6}\right), \gamma(x)>0, \forall x \in \Gamma$. The solution of (6.4) is given by a contraction semi-group $V_{b}(t)=e^{t G_{b}}, t \geq 0$ (see [4] for the definition of $G_{b}$ ) and the spectrum of $G_{b}$ in the half-plan $\{z \in \mathbb{C}: \operatorname{Re} z<0\}$ is formed by isolated eigenvalues with finite multiplicities [2].

We sketch briefly below the similitudes with the analysis in Section 2. If $(E, H) \neq 0$ is an eigenfunction of $G_{b}$ with eigenvalue $\lambda$, then

$$
\left\{\begin{array}{l}
\operatorname{curl} E=-\lambda H, \text { curl } H=\lambda E \text { in } \Omega, \\
\frac{1}{\gamma(x)}(\nu \wedge \nu \wedge E)+\nu \wedge H=0 \text { on } \Gamma, \\
(E, H):(\mathbf{i} \lambda)-\text { outgoing. }
\end{array}\right.
$$

Consider the problem

$$
\left\{\begin{array}{l}
\operatorname{curl} E=-\lambda H, \text { curl } H=\lambda E \text { in } \Omega, \\
\nu \wedge E=f \text { on } \Gamma, \\
(E, H):(\mathbf{i} \lambda)-\text { outgoing. }
\end{array}\right.
$$

In the space $\mathcal{H}_{s}^{t}(\Gamma):=\left\{u \in H^{s}(\Gamma):\langle\nu, u\rangle=0\right\}$ introduce the operator $\mathcal{N}_{b}(\lambda): H_{s+1}^{t}(\Gamma) \rightarrow$ $H_{s}^{t}(\Gamma)$ defined by

$$
\mathcal{N}_{b}(\lambda) f=\left.\nu \wedge H\right|_{\Gamma}
$$

$(E, H)$ being the solution of the problem (6.6). The operator $\mathcal{N}_{b}(\lambda)$ is the analog of the exterior Dirichlet-to-Neumann operator in Section 2 (see [20]) and the boundary condition on (6.5) can be written as

$$
\mathcal{C}_{b}(\lambda) f=\frac{1}{\gamma(x)}(\nu \wedge f)+\mathcal{N}_{b}(\lambda) f=0, f=\left.\nu \wedge E\right|_{\Gamma}
$$

The outgoing resolvent of the problem

$$
\left\{\begin{array}{l}
\operatorname{curl} E=-\lambda H+F_{1}, \operatorname{curl} H=\lambda E+F_{2} \text { in } \Omega \\
\nu \wedge E=0 \text { on } \Gamma \\
(E, H):(\mathbf{i} \lambda)-\text { outgoing }
\end{array}\right.
$$


is analytic for $\operatorname{Re} \lambda<0$ since the above problem corresponds to a self-adjoint operator. Therefor we can prove that $\mathcal{C}_{b}(\lambda)$ is analytic for $\operatorname{Re} \lambda<0$. In the same way from the fact that for $\operatorname{Re} \lambda<0$ there are no non trivial solutions of the problem

$$
\left\{\begin{array}{l}
\operatorname{curl} E=-\lambda H, \operatorname{curl} H=\lambda E \text { in } \Omega, \\
\nu \wedge H=0 \text { on } \Gamma, \\
(E, H):(\mathbf{i} \lambda)-\text { outgoing, }
\end{array}\right.
$$

one concludes that $\mathcal{N}_{b}(\lambda)^{-1}$ is analytic for $\operatorname{Re} \lambda<0$. As in Section 2, one deduces that $\mathcal{C}_{b}(\lambda)^{-1}$ is a meromorphic operator valued function for $\operatorname{Re} \lambda<0$ (see (2.7) and Remark 1). Assuming $\gamma(x) \neq 1, \forall x \in \Gamma$, according to the results in [3], for every $\epsilon>0$ and every $M \in \mathbb{N}, M \geq 1$ the eigenvalues of $G_{b}$ lie in $\Lambda_{\epsilon} \cup \mathcal{R}_{M}$. Next one can establish a trace formula involving $\left(\lambda-G_{b}\right)^{-1}$ and for the analysis of the counting function of the eigenvalue of $G_{b}$ in $\Lambda$ it is possible to apply the strategy of Sections 4,5 combined with the semi-classical parametrix constructed in [20].

\section{ACKNOWLEDGMENTS}

Thanks are due to the referees for their critical comments and useful suggestions leading to an improvement of the previous version of the paper.

\section{REFERENCES}

[1] F. Cakoni and H. Haddar, Transmission eigenvalues in Inverse Scattering Theory. Inside and Out II, MSRI Publications 60, 2012.

[2] F. Colombini, V. Petkov and J. Rauch, Spectral problems for non elliptic symmetric systems with dissipative boundary conditions, J. Funct. Anal. 267 (2014), 1637-1661.

[3] F. Colombini, V. Petkov and J. Rauch, Eigenvalues of the Maxwell's equations with dissipative boundary conditions, Asymptotic Analysis, 99 (2016), 105-124.

[4] F. Colombini and V. Petkov, Weyl formula for the negative dissipative eigenvalues of Maxwell's equations, Archiv der Mathematik, 110 (2018), 183-195.

[5] M. Dimassi and J. Sjöstrand, Spectral asymptotics in semi-classical limits, London Mathematical Society, Lecture Notes Series, 268, Cambridge University Press, 1999.

[6] P. Lax and R. Phillips, Scattering Theory, Second edition, Academic Press, New York, 1989.

[7] P. Lax and R. Phillips, Scattering theory for dissipative systems, J. Funct. Anal. 14 (1973), 172-235.

[8] A. Majda, Disappearing solutions for dissipative wave equation, Indiana Univ. Math. J. 24 (12) (1974), 1119-1133.

[9] A. Majda, The location of the spectrum of the dissipative acoustic operator, Indiana Univ. Math. J. 25 (1976), 973-987.

[10] R. Melrose, Polynomial bound on the distribution of poles in scattering by an obstacle, Journées Equations aux Dérivées Partielles, (1984), 1-8.

[11] Hoai-Minh Nguyen and Quoc-Hung Hguyen, The Weyl law of the transmission eigenvalues and the completeness of generalised transmission eigenfunctions, J. Funct. Anal, 281 (2001), 1091.

[12] V. Petkov, Location of eigenvalues for the wave equation with dissipative boundary conditions, Inverse Probl. Imagining, 10 (2016), 1111-1139.

[13] V. Petkov and G. Vodev, Asymptotics of the number of the interior transmission eigenvalues, J. Spectral Theory, 7 (2017), 1-31.

[14] L. Robiano, Spectral analysis of the interior transmission eigenvalue problem, Inverse Problems, 29, (2013), 104001.

[15] J. Sjöstrand and G. Vodev, Asymptotics of the number of Rayleigh resonances, Math. Ann. 309 (1997), 287-306. 
[16] J. Sjöstrand, Weyl law for semi-classical resonances with random perturbed potentials, Mémoires de SMF, 136 (2014).

[17] G. Vodev, Transmission eigenvalue-free regions. Commun. Math. Phys. 336 (2015), 1141-1166.

[18] G. Vodev, High-frequency approximation of the interior Dirichlet-to-Neumann map and applications to the transmission eigenvalues, Anal. \& PDF, 11 (1) (2018), 213-236.

[19] G. Vodev, Parabolic transmission eigenvalues in the degenerate isotropic case, Asymptotic Analysis, 106 (2018), 147-168.

[20] G. Vodev, Semiclassical parametrix for the Maxwell equation and applications to the electromagnetic transmission eigenvalues, Res. Math. Sci. 8 (3) (2021), Paper 35.

Université de Bordeaux, Institut de Mathématiques de Bordeaux, 351, Cours de la Libération, 33405 Talence, France

Email address: petkov@math.u-bordeaux.fr 